\title{
Arthroscopically Guided Proximal Tibial Epiphysiodesis Screw Placement as Treatment of a Bilateral Partial Cranial Cruciate Ligament Injury in a Juvenile Dog
}

\author{
Mélanie Olive $^{1} \quad$ Alexandre Caron $^{1} \quad$ Alexandre Fournet $^{1} \quad$ Maïa Vanel $^{1}$ \\ ${ }^{1}$ Small Animal Surgery Department, Centre Hospitalier Vétérinaire \\ Atlantia, Nantes, France \\ Address for correspondence Mélanie Olive, DVM, Small Animal \\ Surgery Department, Centre Hospitalier Vétérinaire Atlantia, \\ CHV Atlantia, 22 rue René Viviani, 44200 Nantes, France \\ VCOT Open 2020;3:e112-e118. \\ (e-mail: melanieolive@hotmail.fr).
}

\author{
Abstract \\ Keywords \\ - arthroscopic \\ treatment \\ - epiphysiodesis \\ - cruciate ligament \\ injury \\ - growth plate \\ pathology \\ - juvenile dog
}

This study aimed to describe surgical technique of arthroscopically guided proximal tibial epiphysiodesis screw placement in the treatment of a bilateral partial cranial cruciate ligament $(\mathrm{CrCL})$ rupture in a 5.6-month-old Golden Retriever. A 19-kg, 5.6month-old female Golden Retriever was diagnosed with bilateral partial $\mathrm{CrCL}$ rupture. Proximal tibial epiphysiodesis was performed bilaterally under arthroscopic guidance. Arthroscopic stifle joint inspection was performed bilaterally with a $2.7 \mathrm{~mm}$ arthroscope. A medial port at the level of the distal one-third of the patella was used as camera portal. A lateral instrument port was positioned at the level of the distal onethird of the patella. A $3.0 \mathrm{~mm}$ diameter headless cannulated self-compressive screw was placed into the centre of the tibial cranial intercondyloid area as parallel as possible to the tibial shaft axis under arthroscopic control. Screw head was buried. Arthroscopic guidance allowed good accuracy in screw placement, specifically in its insertion point. However, the screw orientation was less satisfactory. The tibial plateau angle progressively decreased in both stifles to achieve 15 degrees on the left and 16 degrees on the right at the last follow-up. A varus deformity developed on both stifles. No clinical consequences were observed. Recurrence of right pelvic lameness occurred 1 year postoperatively because of a medial meniscal lesion, which necessitated partial caudal meniscectomy. Arthroscopically guided proximal tibial epiphysiodesis is technically feasible and is a minimally invasive treatment of $\mathrm{CrCL}$ injury in a juvenile dog.

\section{Introduction}

Canine cranial cruciate ligament $(\mathrm{CrCL})$ rupture is usually a chronic degenerative disease that leads to partial or complete midsubstance ligament rupture. ${ }^{1}$ Its pathogenesis has not been clearly elucidated, although several risk factors, such as developmental, inflammatory (infectious versus immune-mediated), genetic, metabolic and hormonal pathologies, have been suggested. ${ }^{1,2}$ However, the pathogenesis of CrCL rupture in young dogs is recognized to be more often of traumatic origin. In addition, avulsion of the liga-

received

April 19, 2020

accepted

August 12, 2020
DOI https://doi.org/

$10.1055 / \mathrm{s}-0040-1716821$. ISSN 2625-2325. ment with an osteochondral fragment from the femoral origin or tibial insertion of the $\mathrm{CrCL}$ is commonly observed. ${ }^{1,3-5}$

Several surgical procedures have been proposed for $\mathrm{CrCL}$ rupture treatment in dogs. Among them, tibial plateau levelling techniques, such as tibial plateau levelling osteotomy, allow the neutralization of the cranial tibial thrust by changing the slope of the tibial plateau. ${ }^{2,6,7}$ Although this is a commonly accepted technique for adult dogs, its use for immature dogs is a controversial topic due to the higher risk of complications as well as iatrogenic trauma to the open

(c) 2020 Georg Thieme Verlag KG License terms Stuttgart · New York

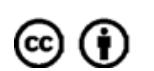


proximal tibial growth plate. ${ }^{4}$ Proximal tibial epiphysiodesis (PTE), which consists of fusing the craniocentral portion of the proximal tibial epiphysis while allowing the continued growth of the caudal aspect of the plateau, was developed in an attempt to progressively level the tibial slope in immature dogs. ${ }^{3,5,8}$ This procedure should be performed prior to proximal tibial epiphyseal closure to allow for significant tibial plateau angle (TPA) changes, and dogs younger than 7 months old have been reported to be the best candidates. 5,8

To our knowledge, only the open approach version of PTE has been reported in the literature. As such, the purpose of this case report is to report the feasibility of arthroscopically guided PTE screw placement.

\section{Case Description}

A $19 \mathrm{~kg}, 5.5$-month-old female Golden Retriever presented with two lameness episodes within 2 months. The episodes involved both pelvic limbs. The dog was referred following a lack of improvement with conservative treatment (strict rest, non-steroidal anti-inflammatory drugs). General physical and neurological assessments were within normal limits. A subjective gait evaluation revealed grade three left pelvic limb lameness, according to the grading scheme published by Monk and colleagues. ${ }^{9}$ The left pelvic limb examination revealed moderate stifle joint effusion with moderate quadriceps muscle atrophy. The cranial tibial compression test was positive without a direct drawer sign or meniscal click. The right pelvic limb examination revealed mild stifle joint effusion as the only abnormality.

Sedation was obtained with methadone $(0.2 \mathrm{mg} / \mathrm{kg}$, intravenous [IV]) and medetomidine ( $3 \mu \mathrm{g} / \mathrm{kg}$ IV). Routine gas anaesthesia was performed.

Mediolateral and craniocaudal orthogonal radiographs of both stifles showed effacement of the infrapatellar fat pad consistent with stifle joint effusion, which was more pronounced on the left side. No radiographic signs of degenerative joint disease were present. The TPA measurements on the mediolateral were 21 and 26 degrees on the left and the right side, respectively, according to the Slocum method., The medial mechanical proximal tibial angle was also calculated on the craniocaudal ${ }^{10,11}$ (-Table $\mathbf{1}$ ).

A computed tomographic (CT) examination of both stifles was performed (contiguous transverse images $[0.625 \mathrm{~mm}$

Table 1 TPA and mMTPA, calculated at each follow-up

\begin{tabular}{|l|l|l|l|l|}
\hline & \multicolumn{2}{|c|}{ TPA } & \multicolumn{2}{c|}{ mPTA } \\
\hline & Left & Right & Left & Right \\
\hline T0 & 21 & 26 & 90 & 96 \\
\hline T0 +1 mo & 16 & 22 & 86 & 91 \\
\hline T0 + 2 mo & 16 & 20 & 85 & 90 \\
\hline T0 +6 mo & 15 & 16 & 85 & 88 \\
\hline T0 + 12 mo & 15 & 16 & 84 & 88 \\
\hline
\end{tabular}

Abbreviations: TPA, tibial plateau angle; mMPTA, medial mechanical proximal tibial angle. thickness], 16 slice CT scanner [GE Brivo CT385, GE Medical Systems, France], bone and soft tissue algorithm and $160 \mathrm{~mA}$ and $130 \mathrm{kV}$ ) (-Fig. 1A). Positive-contrast CT arthrograms were performed with the instillation of $5 \mathrm{~mL}$ of iodixanol (Visipaque 320, GE Healthcare, France, $320 \mathrm{mg} \mathrm{I} / \mathrm{mL}$ ) in each stifle joint. The results revealed a large and bilateral subchondral bone defect at the medial aspect of the lateral femoral condyles associated with moderate-to-severe subchondral sclerosis. Several small mineralized bony fragments were detected at the level of these defects. Partial filling of the subchondral defects was detected bilaterally on the CT arthrography, along with extension of the contrast media into the proximal enthesis of the $\mathrm{CrCL}$, suggesting a partial tear. This was particularly evident on the left side. Based on these findings, an atypical localization of osteochondritis dissecans associated with a partial avulsion of the CrCL was considered the most likely diagnosis (-Fig. 1B). Avulsion of the $\mathrm{CrCL}$ could not be completely ruled out but appeared less likely considering the history, the CT appearance of the bone lesions and the bilateral aspect of the lesions. $^{12}$

Arthroscopic stifle joint inspection was performed on both stifles with a 30-degree $2.7 \mathrm{~mm}$ arthroscope connected to an ultra-high-definition camera system (Synergy UHD4, Arthrex, Munich, Germany). A medial port was created at the level of the distal one-third of the patella and used as a camera portal. Egress was created with a 21-gauge needle in the lateral stifle joint compartment. A fluid pump (Continuous Wave 4, Arthrex, Munich, Germany) was used for stifle joint inflation (50 $\mathrm{mm} \mathrm{Hg}$ ).

The arthroscopic examination showed some degree of CrCL fibrillation in both stifles, which was consistent with a bilateral partial CrCL rupture. Interestingly, a loose (and nonosteochondral) cartilage flap, which was still attached to the ruptured fibre, was observed on the left side. The osteochondral femoral lesions identified on the $\mathrm{CT}$ could not be reached without risking iatrogenic damage on the $\mathrm{CrCL}$ remnants. No meniscal tear was observed on either stifle.

Ruptured $\mathrm{CrCL}$ fibres were debrided with a motorized shaver together with synovial proliferations and a fat pad. A cranial PTE was performed bilaterally via two arthroscopic portals: the medial port for the camera and a lateral port for instrument placement, which was positioned at the distal one-third of the patella and symmetrically to the medial portal. An arthroscopic stainless-steel cannula was used to facilitate portal preservation. It also allowed better medial retraction of the patellar tendon. A $1.2 \mathrm{~mm}$ Kirschner wire was inserted into the centre (mediolaterally) of the tibial cranial intercondyloid area and as cranially as possible into the joint space in the craniocaudal axis from the $\mathrm{CrCL}$ insertion (cranial to the intermeniscal ligament) ( - Fig. 2A). Its orientation into the tibial shaft was as parallel as possible to the mechanical axis of the tibia. A $3.0 \mathrm{~mm}$ diameter headless cannulated self-compressive screw (DePuy Synthes, Saint Priest, France) was manually placed over the guide wire, which was eventually removed (-Fig. 2B, C). The screw head was buried under the arthroscopic control (-Fig. 2D). 


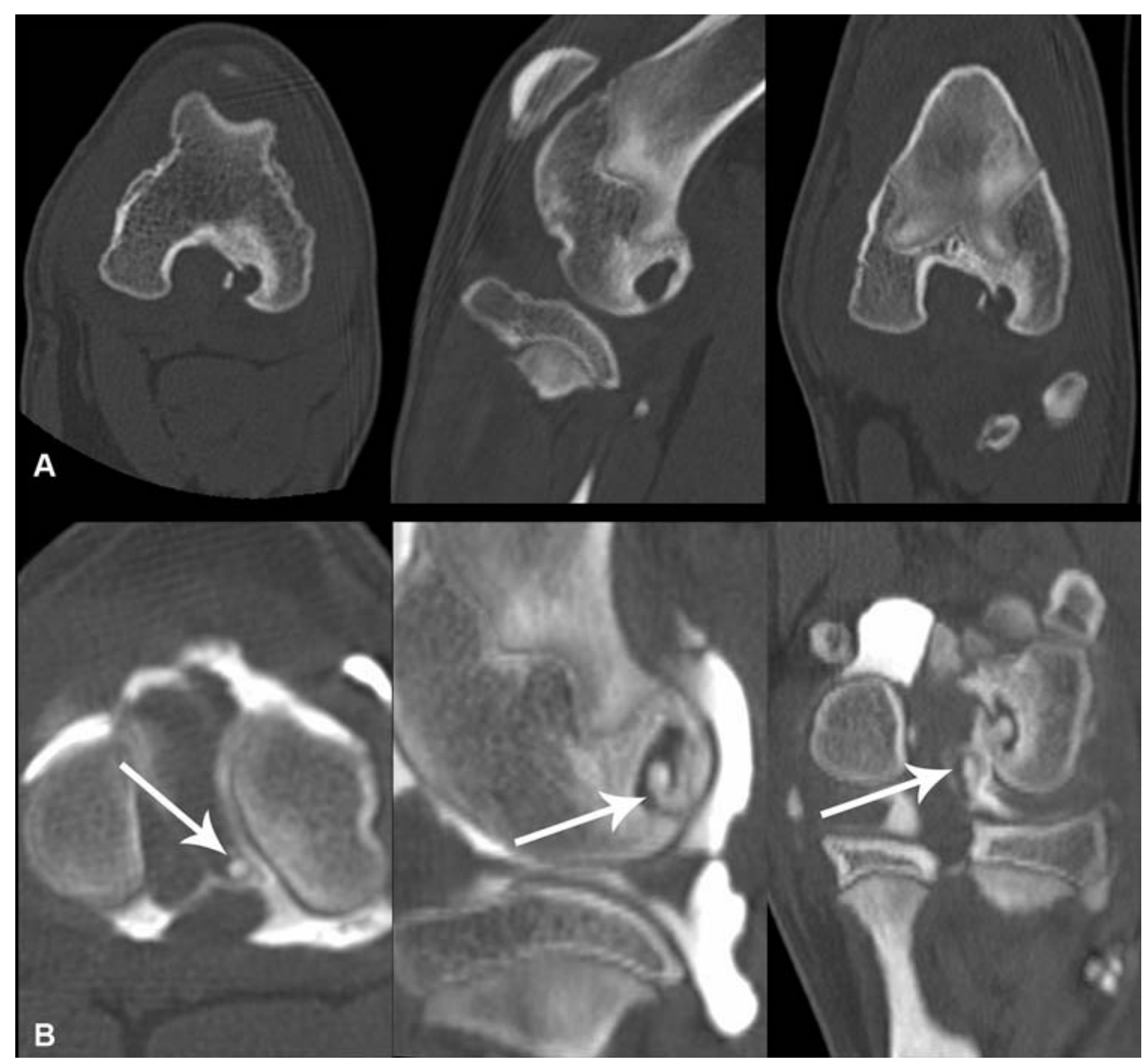

Fig. 1 Preoperative CT scan examination (A) and CT arthrogram (B) images of the left stifle in transverse, sagittal and frontal reconstructions. (A) A large subchondral bone defect can be observed at the medial aspect of the lateral femoral condyle associated with subchondral bone sclerosis. (B) Contrast media can be detected within the proximal aspect of the cranial cruciate ligament, indicating a partial tear; however, ligament thickness is normal. White arrow represents an antypical localization of osteochondritis dissecans associated with a partial avulsion of the $\mathrm{CrCL}$ that was considered the most likely diagnosis. $\mathrm{CrCL}$, canine cranial cruciate ligament; $\mathrm{CT}$, computed tomography.

The postoperative CT scan confirmed that the screw insertion points were correct. However, the screw orientation was less satisfactory; the screw on the left side was placed 10 degrees caudal and 12 degrees medial relative to the tibial mechanical axis, and the screw on the right side was placed 7 degrees cranial and 12 degrees medial relative to the tibial mechanical axis ( $\mathbf{- F i g s .} 3$ and $\mathbf{4}$ ). The screw tip on the right side exited slightly across the medial cortex.

Amoxicillin/clavulanic acid was administered $(12.5 \mathrm{mg} / \mathrm{kg}$ IV) peri- and postoperatively every 12 hours for 24 hours. Analgesia was provided with meloxicam $(0.2 \mathrm{mg} / \mathrm{kg}$ subcutaneous q24h) and methadone $(0.2 \mathrm{mg} / \mathrm{kg}$ IV q4h) for 24 hours postoperatively. The dog was discharged 24 hours after surgery with a 2-week course of meloxicam $(0.1 \mathrm{mg} / \mathrm{kg}$ per os [PO] q24h) and amoxicillin/clavulanic acid (12.5 mg/kg PO q12h) due to superficial pyodermatitis at the time of surgery. Leashonly walking was recommended for 6 weeks.

The left pelvic limb lameness progressively improved. At the 2-month follow-up, the orthopaedic examination on the right side remained unremarkable. Three months after surgery, no lameness was observed on either pelvic limb.

The radiographic examination at 1, 2 and 6 months after surgery revealed a progressive decrease in TPA to achieve
15 degrees on the left and 16 degrees on the right at the last follow-up (indicating 6 and 10 degrees improvement, respectively, relative to the preoperative radiographs). A varus deformity of 6 degrees on the left stifle and 8 degrees and the right stifle was also observed ( - Table 1; - Fig. 4).

One year postoperatively, the owners reported a sudden recurrence of right pelvic limb lameness. The orthopaedic examination showed moderate right thigh muscle atrophy and stifle effusion. A meniscal click was also noted. A cranial drawer sign was present with a negative tibial compression test. On the right stifle radiographs, stifle joint effusion was observed; the osteophytosis had not progressed.

Right stifle arthroscopy revealed a complete $\mathrm{CrCL}$ rupture with a full-thickness vertical longitudinal tear of the caudal horn of the medial meniscus. Therefore, an arthroscopic partial caudal medial meniscectomy was performed. The PTE screw was also removed under arthroscopic guidance. Since the distal tip of the screw protruded through the medial cortex, a guide wire was introduced from distal to proximal, allowing easy guidance of the screwdriver.

Lameness improved substantially over the first 2 weeks, and no lameness was observed 3 months after meniscectomy. At the last follow-up ( 5 months after the last surgery 


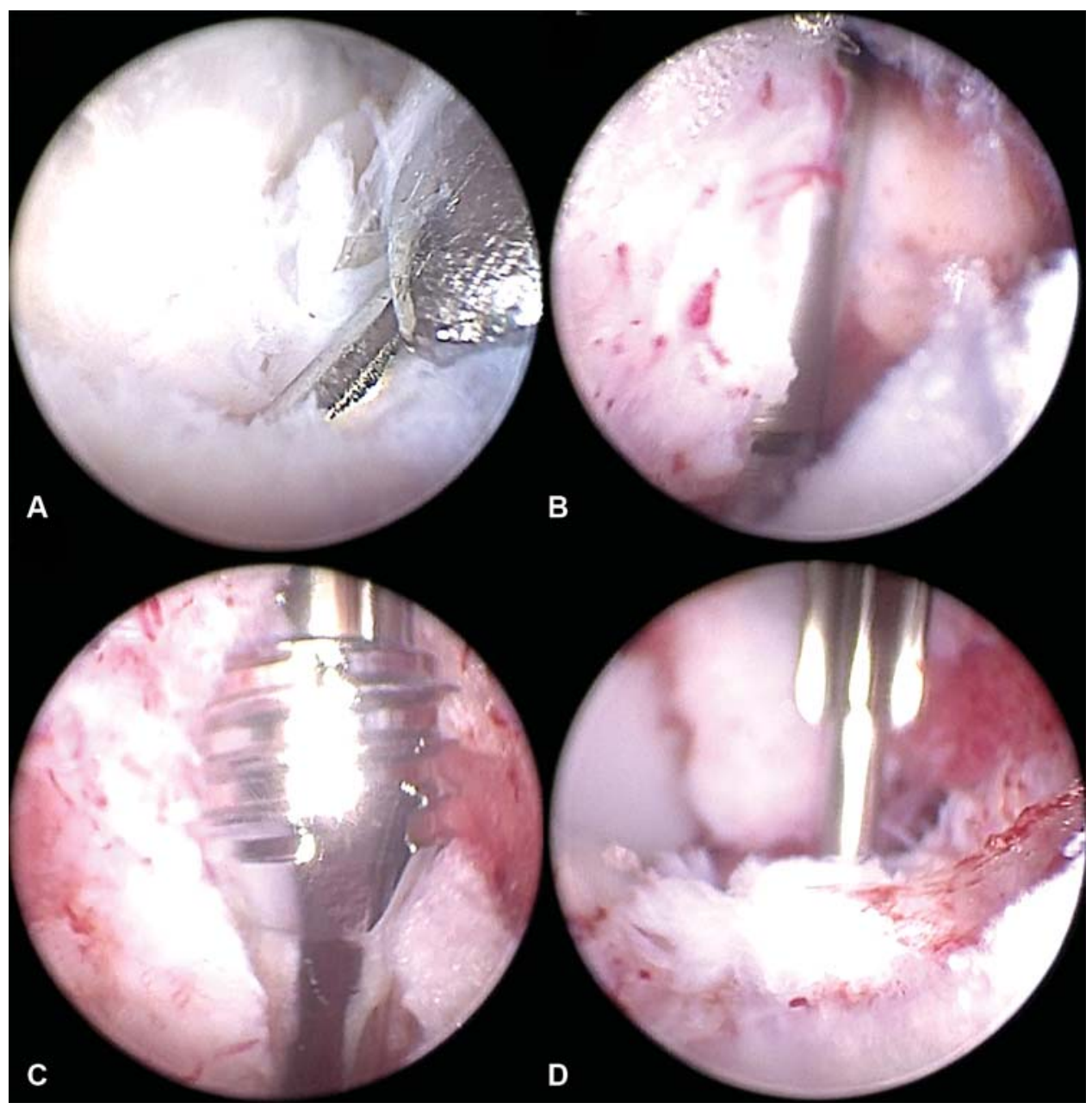

Fig. 2 Arthroscopic still images of stifle proximal tibial epiphysiodesis screw placement (left stifle: camera in the medial portal). (A) Arthroscopic view of the Kirschner wire through the cannula in place on the tibial plateau, just cranial to the transverse ligament. Cranial cruciate ligament insertion is just behind the instruments. (B) Arthroscopic view of the cannulated drill bit and its measuring marks while over drilling (C) Arthroscopic view of the top of the $3.0 \mathrm{~mm}$ headless self-compressive screw while manually screwing. Note the threaded self-tapping screw head. (D) Arthroscopic view of the screw placement site after burying the screw and while removing the screwdriver.

and 16 months after bilateral PTE), no lameness was observed on either hindlimbs. The orthopaedic examination was unremarkable; specifically, no stifle effusion was palpated, and the tibial compression test was negative, bilaterally.

\section{Discussion}

This report describes arthroscopically guided PTE in the treatment of a bilateral partial CrCL injury in a juvenile dog. Treatment of CrCL avulsion or tears in juvenile dogs can be challenging. ${ }^{13}$ Proximal tibial epiphysiodesis has been described as the sole treatment to level the TPA in skeletally immature dogs with CrCL-deficient stifle joints. ${ }^{13-15}$ In a previous report, intraoperative fluoroscopic assessment ensured appropriate screw placement via an open approach. ${ }^{5}$ Our case report describes the first PTE screw placement in a dog using arthroscopic guidance, with good accuracy in screw placement, specifically in its insertion point.
Arthroscopy is a commonly performed procedure in veterinary orthopaedic surgery. Compared with arthrotomy, the documented advantages of this minimally invasive procedure include lower morbidity with quicker return to normal function, decreased complication rates and improved functional outcomes. ${ }^{16,17}$ Furthermore, the magnification provided by arthroscopy allows a superior examination of anatomical structures and instrument placement precision. ${ }^{18}$ As such, it enables a more precise diagnosis of intraarticular disease. In an ex vivo study, arthroscopy was more accurate than arthrotomy in diagnosing meniscal lesions in a canine stifle joint. ${ }^{17}$ Thus, we considered arthroscopy as an interesting alternative for PTE compared with the open approach described by Vezzoni and colleagues. ${ }^{5}$ However, this procedure has several disadvantages. Rogatko and colleagues demonstrated that iatrogenic articular cartilage injury was greater in dogs after stifle joint arthroscopy than after mini-arthrotomy. ${ }^{19}$ Owen and colleagues reported that radiographic patellar thickening was more frequent following arthroscopy than arthrotomy. ${ }^{20}$ There were no 


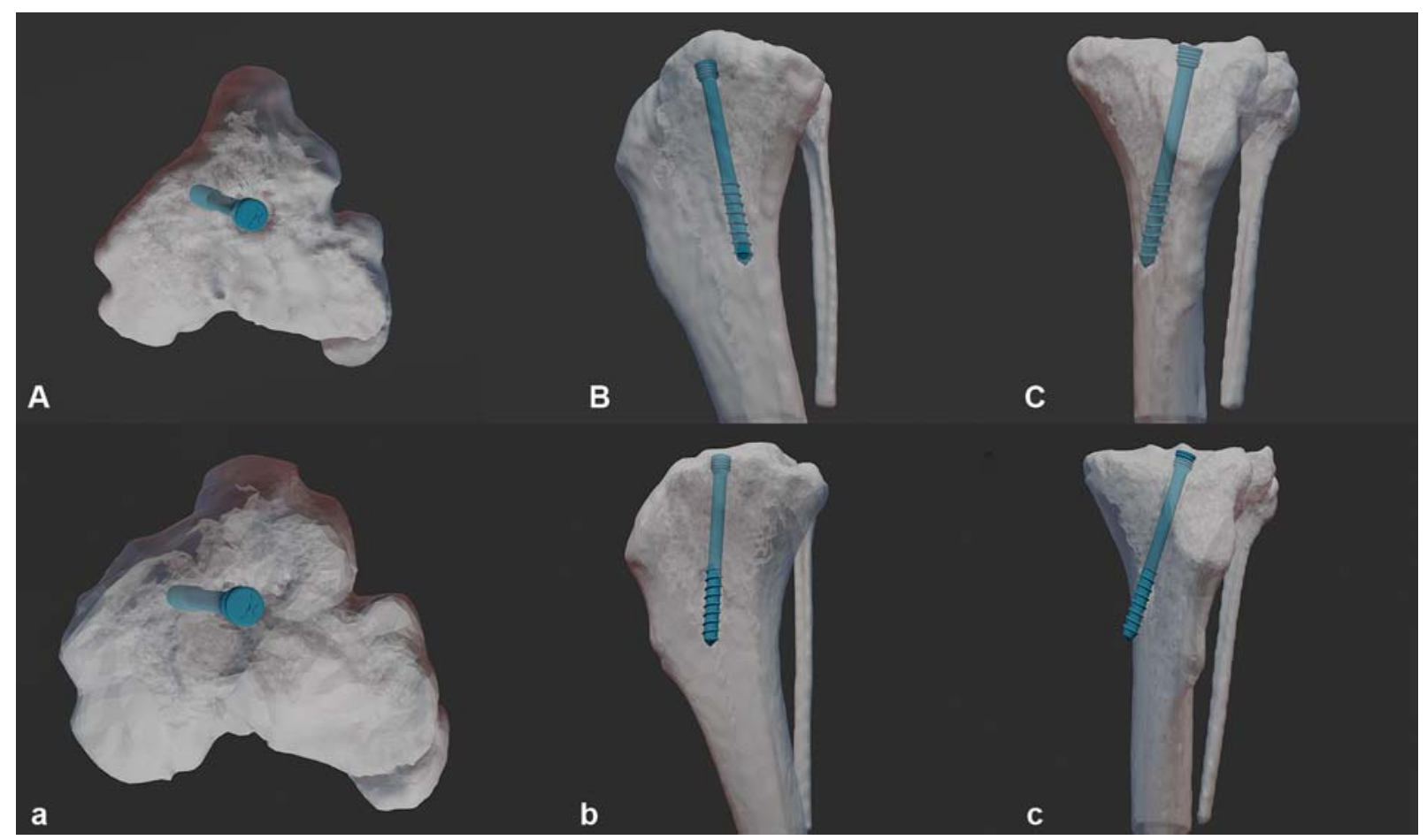

Fig. 3 Postoperative three-dimensional reconstruction images of the left (A, B, C) and right (a, b, C) stifles in transverse (A, a), sagittal (B, b) and frontal $(C, C)$ planes, showing the screw position and orientation.

radiographic signs of patellar tendon thickening at any time in the present case. Proximal tibial epiphysiodesis screw insertion is a surgery of the cranial stifle joint compartment, and previous studies have shown that surgeries in this compartment result in less iatrogenic articular cartilage injury. ${ }^{19,20}$ As this is a case report, further larger ex vivo and in vivo studies are required to identify whether the arthroscopic or open approach for PTE placement results in less morbidity.

The PTE principle is that fusion of the craniocentral portion of the proximal tibial epiphysis ceases growth while allowing continued growth of the caudal aspect of the plateau. ${ }^{3}$ This is permitted by placing a screw into the tibial axis that necessitates patellar tendon retraction without patellar luxation. In their open approach, Vezzoni and colleagues used a Gelpi retractor after a 2 to $3 \mathrm{~cm}$ parapatellar mini-arthrotomy. ${ }^{5}$ In our case, a $3 \mathrm{~mm}$ portal, which was lateral to the patellar tendon, was used for screw positioning, leading to less periarticular tissue laxity but a more difficult patellar tendon retraction. Orienting the guide wire parallel to the tibial axis was much more challenging, which could explain the medial orientation of our screws. Fluoroscopic guidance, as described in a previous report, ${ }^{5}$ would have allowed better control of this screw angulation, but it was not available in our clinic.

Vezzoni and colleagues reported angular deformity, especially valgus deformity of the tibia, as a complication of PTE in 3 of 22 cases. $^{5}$ For the authors, the screw insertion point seemed to be critical to avoid this complication. ${ }^{5}$ In our case, the chosen arthroscopic landmarks appeared to be accurate, as confirmed by advanced postoperative diagnostic imaging.
Further studies in a larger population and involving multiple surgeons are still required to evaluate the precision and accuracy of PTE screw placement. Despite an accurate screw insertion point, a varus deformity of 6 degrees on the left stifle and 8 degrees on the right stifle was observed, although no clinical consequences were observed. Therefore, the screw angulation into the tibial shaft could also influence postoperative tibial deformation.

Several studies have been conducted on the growth potential of young Labrador Retrievers. ${ }^{3,21}$ These studies agreed that the greatest growth rate was between 4 and 5 months old. It decreased until the 13th month with little growth $(<2 \mathrm{~mm})$ noted monthly after 10 months. ${ }^{3,21}$ As such, it is critical to perform TPE as early as possible in young dogs to achieve sufficient TPA correction. In the present case, the dog was 5.5 months old, and the authors did not delay the procedure despite the presence of a cutaneous lesion at the time of surgery. McBrien and colleagues published a curve that predicts TPA modification obtained after PTE. ${ }^{3}$ Based on that study, TPA would have been equal to 10.4 degrees on the right side and 8.4 degrees on the left side, which is a higher correction than those obtained in the present case. Interspecies and interindividual growth rate variability should therefore be considered. ${ }^{13,22}$

Progression to complete $\mathrm{CrCL}$ rupture associated with a medial meniscal lesion in the right stifle was documented 1 year after surgery. This could be explained by the persistence of femorotibial instability, since our postoperative TPA was, according to Hulse and colleagues, insufficient to transform cranial tibial thrust to caudal tibial thrust and, thus, insufficient to protect the meniscus from a postliminary 

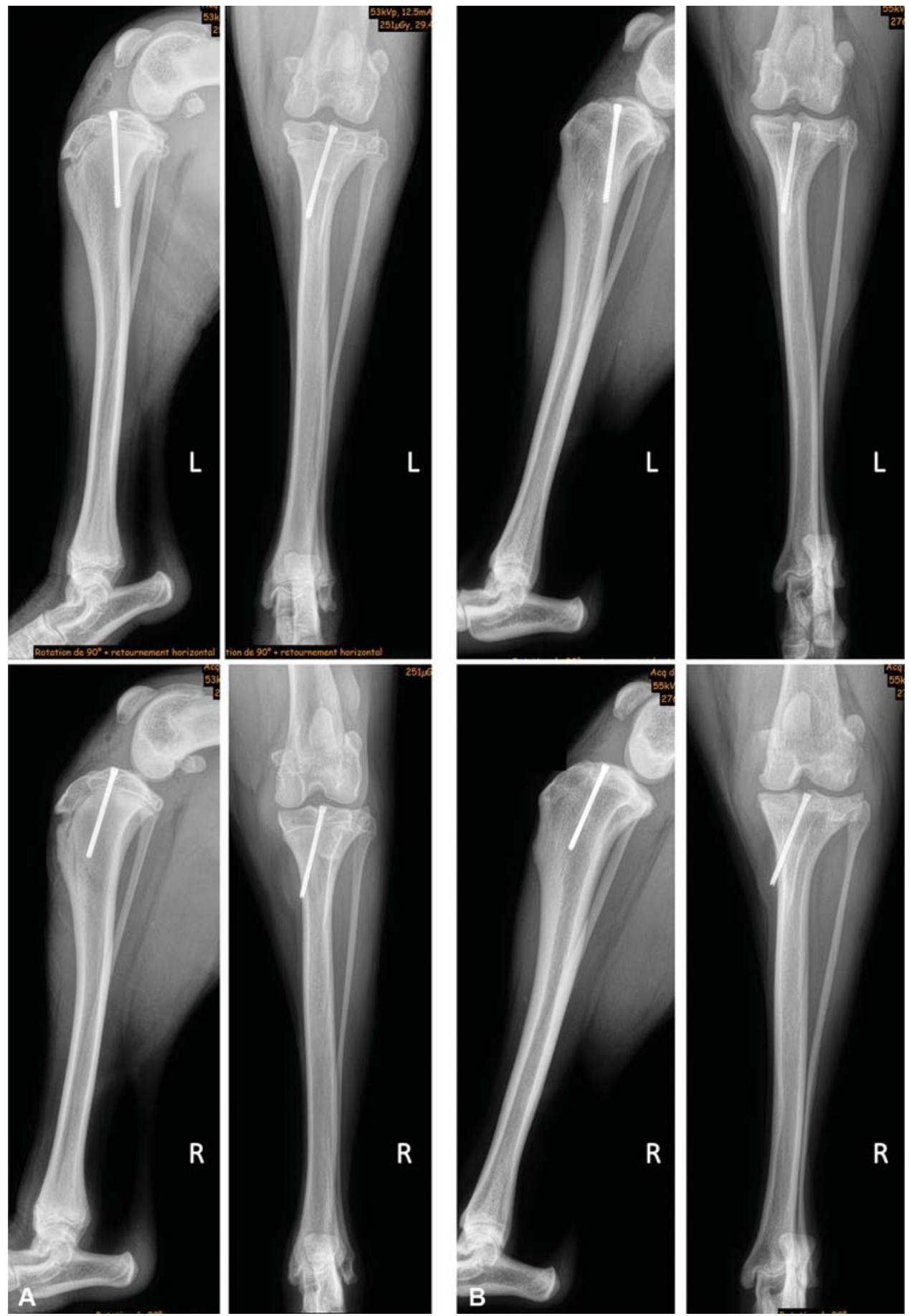

Fig. 4 Postoperative bilateral sequential orthogonal radiographic follow-up to assess tibial plateau angle (TPA) changes and absence of bone deformity development. (A) Postoperative radiographic examination. The TPA measurements are 26 degrees on the right and 21 degrees on the left. Note the mediolateral and craniocaudal screw positioning onto the tibial plateau with the slight mediodistal orientation of both screws (10 and 8 degrees). (B) One-year postoperative radiographic examination. The TPA measurements are 16 degrees on the right and 15 degrees on the left. The tibial growth plates are closed bilaterally.

meniscal tear and the remaining $\mathrm{CrCL}$ from a complete rupture. ${ }^{23}$ The varus deformity which developed on both stifles after the PTE procedure could have also promoted development of a 'pivot shift', facilitating excessive pressure on the medial compartment, and leading to medial meniscal lesion. ${ }^{24}$ Levelling the TPA at the closure of the growth plate, associated with a medial opening crescentic osteotomy, could have improved stifle stability and limited the risk of a subsequent meniscal tear. The dog clinically recovered, muscle mass returned to normal limits and postoperative TPA was similar to the one considered acceptable by Robinson and colleagues, which led to not consider another levelling procedure necessary. ${ }^{25}$ Interestingly, Vezzoni and colleagues only performed additional procedures after PTE in 
two cases (tibial plateau levelling osteotomy for additional tibial plateau levelling and an extraarticular suture for persistent rotational instability). Tibial plateau levelling osteotomy was only considered if persistent lameness after PTE remained. ${ }^{5}$

To our knowledge, this is the first documented case of arthroscopically guided PTE screw placement. This report shows that arthroscopically guided PTE is technically feasible and can be considered in the treatment of $\mathrm{CrCL}$ injury in juvenile dogs. As iatrogenic frontal plane malalignment occurred in both stifles treated, further studies with a higher number of patients are needed to evaluate the accuracy in screw positioning with the technique developed in this case report. Furthermore, these studies could also evaluate the biological benefits (cartilage lesion and patellar tendon damage) and confirm the clinical benefits in terms of postoperative pain and return to normal function after screw placement with arthroscopic guidance instead of the open approach.

Conflict of Interest

None declared.

\section{References}

1 Johnston SA, Tobias KM Stifle joint. In: Veterinary Surgery Small Animal. 2nd edition St Louis, MO: Elsevier; 2018:1071-1168

2 Cook JL. Cranial cruciate ligament disease in dogs: biology versus biomechanics. Vet Surg 2010;39(03):270-277

3 McBrien CS Jr, Vezzoni A, Conzemius MG. Growth dynamics of the canine proximal tibial physis. Vet Surg 2011;40(04):389-394

4 Putame G, Terzini M, Bignardi C, et al. Surgical treatments for canine anterior cruciate ligament rupture: assessing functional recovery through multibody comparative analysis. Front Bioeng Biotechnol 2019;7:180

5 Vezzoni A, Bohorquez Vanelli A, Modenato M, Dziezyc J, Devine Slocum T. Proximal tibial epiphysiodesis to reduce tibial plateau slope in young dogs with cranial cruciate ligament deficient stifle. Vet Comp Orthop Traumatol 2008;21(04):343-348

6 Slocum B, Slocum TD. Tibial plateau leveling osteotomy for repair of cranial cruciate ligament rupture in the canine. Vet Clin North Am Small Anim Pract 1993;23(04):777-795

7 Tinga S, Kim SE, Banks SA, et al. Femorotibial kinematics in dogs treated with tibial plateau leveling osteotomy for cranial cruciate ligament insufficiency: an in vivo fluoroscopic analysis during walking. Vet Surg 2020;49(01):187-199

8 Mesquita LR, Muzzi LAL, Giannico AT, et al. Proximal tibial epiphysiodesis in a growing dog. Acta Sci Vet 2016;44:113-117

9 Monk ML, Preston CA, McGowan CM. Effects of early intensive postoperative physiotherapy on limb function after tibial plateau leveling osteotomy in dogs with deficiency of the cranial cruciate ligament. Am J Vet Res 2006;67(03):529-536
10 Hoelzler MG, Millis DL, Francis DA, Weigel JP. Results of arthroscopic versus open arthrotomy for surgical management of cranial cruciate ligament deficiency in dogs. Vet Surg 2004;33 (02):146-153

11 Johnston SA, Tobias KM Principles of angular limb deformity correction. In: Veterinary Surgery Small Animal. 2nd edition St Louis, MO: Elsevier; 2018:762-774

12 Kulendra E, Lee K, Schoeniger S, Moores AP. Osteochondritis dissecans-like lesion of the intercondylar fossa of the femur in a dog. Vet Comp Orthop Traumatol 2008;21(02):152-155

13 Duval JM, Budsberg SC, Flo GL, Sammarco JL. Breed, sex, and body weight as risk factors for rupture of the cranial cruciate ligament in young dogs. J Am Vet Med Assoc 1999;215(06):811-814

14 Fossum TW, Hedlund CS, Johnson AL, et al. Small Animal Surgery. 3rd edition St Louis, MO: Mosby Elsevier; 2007:1266-1267

15 Brienza PD, Muzzi LAL, Lacreta A Jr, Alves EGL, Guimaraes GC, Muzzi RAL. Electrocautery technique for proximal tibial epiphysiodesis in Labrador retriever puppies: experimental study. Arq Bras Med Vet Zootec 2016;68:1586-1594

16 Johnston SA, Tobias KM. Arthroscopy. In: Veterinary Surgery Small Animal. 2nd edition St Louis, MO: Elsevier; 2018: 1323-1347

17 Pozzi A, Hildreth BE III, Rajala-Schultz PJ. Comparison of arthroscopy and arthrotomy for diagnosis of medial meniscal pathology: an ex vivo study. Vet Surg 2008;37(08):749-755

18 McGinty JB, Burkhart SS. Arthroscopy of the wrist: introduction and indications. In: Operative Arthroscopy. 3rd edition Philadelphia, PA: Lippincott Williams and Wilkins; 2003:721-728

19 Rogatko CP, Warnock JJ, Bobe G, Verpaalen VD. Comparison of iatrogenic articular cartilage injury in canine stifle arthroscopy versus medial parapatellar mini-arthrotomy in a cadaveric model. Vet Surg 2018;47:06-014

20 Owen DJ, Manley R, Casale SA. Influence of methods of joint inspection during tibial plateau leveling osteotomy on the radiographic appearance of the patellar tendon. Vet Surg 2018;47(07): 916-922

21 Odders JW, Jessen CR, Lipowitz AJ. Sequential measurements of the tibial plateau angle in large-breed, growing dogs. Am J Vet Res 2004;65(04):513-518

22 Wordeman SC, Quatman CE, Kaeding CC, Hewett TE. In vivo evidence for tibial plateau slope as a risk factor for anterior cruciate ligament injury: a systematic review and meta-analysis. Am J Sports Med 2012;40(07):1673-1681

23 Hulse D, Beale B, Kerwin S. Second look arthroscopic findings after tibial plateau leveling osteotomy. Vet Surg 2010;39(03): 350-354

24 Fitzpatrick N, Johnson J, Hayashi K, Girling S, Yeadon R. Tibial plateau leveling and medial opening crescentic osteotomy for treatment of cranial cruciate ligament rupture in dogs with tibia vara. Vet Surg 2010;39(04):444-453

25 Robinson DA, Mason DR, Evans R, Conzemius MG. The effect of tibial plateau angle on ground reaction forces 4-17 months after tibial plateau leveling osteotomy in Labrador Retrievers. Vet Surg 2006;35(03):294-299 